\title{
Attacks on the Faithfulness of the Burau Representation of the Braid Group $B_{4}$
}

\author{
Mohammad Y. Chreif ${ }^{1} \&$ Mohammad N. Abdulrahim $^{1}$ \\ ${ }^{1}$ Department of Mathematics, Beirut Arab University, P.O. Box 11-5020, Beirut, Lebanon \\ Correspondence: Mohammad N. Abdulrahim, Department of Mathematics, Beirut Arab University, P.O. Box 11-5020, \\ Beirut, Lebanon. E-mail: mna@bau.edu.lb
}

Received: November 11, 2015 Accepted: December 7, 2015 Online Published: January 7, 2016

doi:10.5539/jmr.v8n1p5 URL: http://dx.doi.org/10.5539/jmr.v8n1p5

\begin{abstract}
The faithfulness of the Burau representation of the 4-strand braid group, $B_{4}$, remains an open question. In this work, there are two main results. First, we specialize the indeterminate $t$ to a complex number on the unit circle, and we find a necessary condition for a word of $B_{4}$ to belong to the kernel of the representation. Second, by using a simple algorithm, we will be able to exclude a family of words in the generators from belonging to the kernel of the reduced Burau representation.
\end{abstract}

Keywords: braid group, Burau representation, faithful

\section{Introduction}

Magnus and Peluso (1969) showed that the Burau representation is faithful for $n \leq 3$. Moody (1991) showed that it is not faithful for $n \geq 9$; this result was improved to $n \geq 6$ by Long and Paton (1992). The non-faithfulness for $n=5$ was shown by Bigelow (1999). The question of whether or not the Burau representation for $n=4$ is faithful is still open.

In our work, we attack the question of faithfulness of the Burau representation of $B_{4}$. In section 3 , we specialize the indeterminate $t$ to a complex number $e^{i \alpha}$, where $\alpha \in \mathbb{R}$. Then we show that if $\frac{\alpha}{\pi} \notin \mathbb{Q}$ and $4 \epsilon+3 m \neq 0$, then the word $b^{\epsilon_{1}} a^{m_{1}} b^{\epsilon_{2}} a^{m_{2}} \ldots \ldots . . b^{\epsilon_{n}} a^{m_{n}}$ does not belong to the kernel of the representation . Here $\epsilon_{i}=0,1$ or $2, m_{i} \in \mathbb{Z}, \epsilon=\sum_{i=1}^{n} \epsilon_{i}$ and $m=\sum_{i=1}^{n} m_{i}$, for $1 \leq i \leq n$. In section 4 , we let $a=\sigma_{1} \sigma_{2} \sigma_{3}$ and $b=a \sigma_{1}$, where $\sigma_{1}, \sigma_{2}, \sigma_{3}$ are generators of $B_{4}$. Then we find the general form of the words $a^{n}$ and $b^{n}$ and we prove that they are not in the kernel of the representation for any non-zero natural number $n$. In section 5, we introduce a simple algorithm which computes all words of the form $a^{i} b^{j}$ and $a^{i} b^{j} a^{k}$ for integers $i, j$ and $k$. We then conclude that there is no word of such forms in the kernel of the representation.

\section{Preliminaries}

Definition 1. (Artin, 1965) The braid group, $B_{n}$, is an abstract group generated by $\sigma_{1}, \sigma_{2}, \ldots, \sigma_{n-1}$ with the following relations

$$
\begin{gathered}
\sigma_{i} \sigma_{j}=\sigma_{j} \sigma_{i}, \text { for all } i, j=1, \ldots, n-1 \text { with }|i-j| \geq 2, \\
\text { and } \\
\sigma_{i} \sigma_{i+1} \sigma_{i}=\sigma_{i+1} \sigma_{i} \sigma_{i+1}, \text { for } i=1, \ldots, n-2 .
\end{gathered}
$$

Definition 2. (Burau, 1936) The reduced Burau representation of $B_{n}$ is defined by

$$
\begin{gathered}
\alpha_{n}: B_{n} \rightarrow G L\left(n-1, \mathbb{Z}\left[t, t^{-1}\right]\right) \\
\sigma_{1}=\left(\begin{array}{ccc}
-t & 1 & 0 \\
0 & 1 & 0 \\
0 & 0 & I_{n-3}
\end{array}\right), \sigma_{n-1}=\left(\begin{array}{ccc}
I_{n-3} & 0 & 0 \\
0 & 1 & 0 \\
0 & t & -t
\end{array}\right), \\
\sigma_{i}=\left(\begin{array}{ccccc}
I_{i-2} & 0 & 0 & 0 & 0 \\
0 & 1 & 0 & 0 & 0 \\
0 & t & -t & 1 & 0 \\
0 & 0 & 0 & 1 & 0 \\
0 & 0 & 0 & 0 & I_{n-(i+2)}
\end{array}\right) \text { where } 2 \leq i \leq n-2 .
\end{gathered}
$$


In particular, setting $n=4$, we have

$$
\sigma_{1}=\left(\begin{array}{ccc}
-t & 1 & 0 \\
0 & 1 & 0 \\
0 & 0 & 1
\end{array}\right), \sigma_{2}=\left(\begin{array}{ccc}
1 & 0 & 0 \\
t & -t & 1 \\
0 & 0 & 1
\end{array}\right) \text { and } \sigma_{3}=\left(\begin{array}{ccc}
1 & 0 & 0 \\
0 & 1 & 0 \\
0 & t & -t
\end{array}\right)
$$

where $t$ is an indeterminate.

Let $a=\sigma_{1} \sigma_{2} \sigma_{3}$ and $b=a \sigma_{1}$. Then we get

$$
a=\left(\begin{array}{ccc}
0 & 0 & -t \\
t & 0 & -t \\
0 & t & -t
\end{array}\right) \text { and } b=\left(\begin{array}{ccc}
0 & 0 & -t \\
-t^{2} & t & -t \\
0 & t & -t
\end{array}\right)
$$

Since the determinants $\left|\sigma_{1}\right|=\left|\sigma_{2}\right|=\left|\sigma_{3}\right|=-t$, it follows that $|a|=-t^{3}$ and $|b|=t^{4}$.

Theorem 1. (Holtzma, 2008) Let $B_{4}$ be the braid group of order 4 then

1. $B_{4}=<a, b \mid a^{4}=b^{3}, a^{2} b a b=b a b a^{2}>$,

2. $Z\left(B_{4}\right)=<a^{4}>$.

Using Theorem 1, we can show that the elements of $B_{4}$ are either of the form $b^{\epsilon_{1}} a^{m_{1}} b^{\epsilon_{2}} a^{m_{2}} \ldots \ldots . . . b^{\epsilon_{n}} a^{m_{n}}$ or of the form obtained by permuting $a^{m_{i}}$ and $b^{\epsilon_{i}}$. Here $\epsilon_{i}=0,1,2$ and $m_{i} \in \mathbb{Z}(i=1, \ldots, n)$.

\section{Necessary Condition for Elements in the Kernel of the Reduced Burau Representation}

Let $t$ be a non-zero complex number on the unit circle, $t=e^{i \alpha}$, where $\alpha$ is a non-zero real number.

Theorem 2. Given a non zero integer $n$ and a word $u$ in $B_{4}, \epsilon_{i}=0,1$ or $2, m_{i} \in \mathbb{Z}, \epsilon=\sum_{i=1}^{n} \epsilon_{i}$ and $m=\sum_{i=1}^{n} m_{i}$. Suppose that $\frac{\alpha}{\pi} \notin \mathbb{Q}$. If $u$ is a non empty word of the form $b^{\epsilon_{1}} a^{m_{1}} b^{\epsilon_{2}} a^{m_{2}} \ldots . . . b^{\epsilon_{n}} a^{m_{n}}$ such that $4 \epsilon+3 m \neq 0$, then $u$ does not belong to the kernel of the representation.

Proof. $|u|=|b|^{\epsilon}|a|^{m}=1$. So $(-1)^{m} t^{4 \epsilon+3 m}=1$. Then we have 2 cases.

(i) If $m$ is even then $e^{i \alpha(4 \epsilon+3 m)}=1$ and so $(4 \epsilon+3 m) \alpha=2 k \pi$, where $k \in \mathbb{Z}$. This implies that $\frac{\alpha}{\pi} \in \mathbb{Q}$, which is a contradiction.

(ii) If $m$ is odd then $e^{i \alpha(4 \epsilon+3 m)}=-1$ and so $(4 \epsilon+3 m) \alpha=(2 k+1) \pi$, where $k \in \mathbb{Z}$. This implies that $\frac{\alpha}{\pi} \in \mathbb{Q}$, which is a contradiction.

Likewise for a word obtained from $u$ by permuting $b^{\epsilon_{i}}$ and $a^{m_{i}}$.

Corollary 1. For a word $u$ of the form $b^{\epsilon_{1}} a^{m_{1}} b^{\epsilon_{2}} a^{m_{2}} \ldots \ldots . . b^{\epsilon_{n}} a^{m_{n}}$ to belong to the kernel of the representation, $m$ has to belong to $4 \mathbb{Z}$ and $\epsilon$ has to belong to $3 \mathbb{Z}$.

\section{The Words $a^{n}$ and $b^{n}$}

In this section, we find the general form of the words $a^{n}$ and $b^{n}$, for any integer $n$. Denote by $I_{3}$ the identity matrix. We recall, from section 2, that $a=\sigma_{1} \sigma_{2} \sigma_{3}$ and $b=a \sigma_{1}$. It is easy to see that $b^{-1}=b^{2} a^{-4}$ and $b^{-2}=b a^{-4}$.

Proposition 1. Consider the matrix $J=\left(\begin{array}{ccc}0 & 0 & -1 \\ 1 & 0 & -1 \\ 0 & 1 & -1\end{array}\right)$. For any $k \in \mathbb{N}$, we have

$$
\begin{aligned}
& \text { 1. } a^{4 k}=t^{4 k} I_{3} \text {, } \\
& a^{4 k+2}=t^{4 k+2} J^{2} \text {, } \\
& a^{4 k+1}=t^{4 k+1} J, \\
& a^{4 k+3}=t^{4 k+3} J^{3}, \\
& a^{-(4 k+1)}=t^{-(4 k+1)} J^{3}, \\
& a^{-(4 k+3)}=t^{-(4 k+3)} \mathrm{J} \text {. }
\end{aligned}
$$

Proof. We prove this proposition using mathematical induction principle. For $k=0$ and $k=1$, direct computations give us that $J^{4}=1$, 


$$
\begin{aligned}
& a^{0}=I_{3}, a^{1}=t J, a^{2}=t^{2} J^{2}, a^{3}=t^{3} J^{3}, \\
& a^{4}=t^{4} I_{3}, a^{5}=t^{5} J, a^{6}=t^{6} J^{2}, a^{7}=t^{7} J^{3}, \\
& a^{-1}=t^{-1} J^{3}, a^{-2}=t^{-2} J^{2}, a^{-3}=t^{-3} J, a^{-4}=t^{-4} I_{3},
\end{aligned}
$$

and

$$
a^{-5}=t^{-5} J^{3}, a^{-6}=t^{-6} J^{2}, a^{-7}=t^{-7} J
$$

Suppose that the proposition is true for all integers less than or equal to $k$. We then show it is still true for $k+1$. We show (1):

$$
\begin{aligned}
& a^{4(k+1)}=a^{4 k} a^{4}=t^{4 k} I_{3}=t^{4(k+1)} I_{3} \\
& a^{4(k+1)+1}=a^{4 k} a^{5}=t^{4 k} I_{3} t^{5} J=t^{4(k+1)+1} J \\
& a^{4(k+1)+2}=a^{4 k} a^{6}=t^{4 k} I_{3} t^{6} J^{2}=t^{4(k+1)+2} J^{2} \\
& a^{4(k+1)+3}=a^{4 k} a^{7}=t^{4 k} I_{3} t^{7} J^{3}=t^{4(k+1)+3} J^{3}
\end{aligned}
$$

We show (2):

$$
\begin{aligned}
& a^{-4(k+1)}=a^{-(4 k)} a^{-4}=t^{-(4 k)} I_{3} t^{-4} I_{3}=t^{-4(k+1)} I_{3} \\
& a^{-(4(k+1)+1)}=a^{-4 k} a^{-5}=t^{-4 k} I_{3} t^{-5} J^{3}=t^{-(4(k+1)+1)} J^{3} \\
& a^{-(4(k+1)+2)}=a^{-4 k} a^{-6}=t^{-4 k} I_{3} t^{-6} J^{2}=t^{-(4(k+1)+2)} J^{2} \\
& a^{-(4(k+1)+3)}=a^{-4 k} a^{-7}=t^{-4 k} I_{3} t^{-7} J=t^{-(4(k+1)+3)} J
\end{aligned}
$$

Therefore the proposition is true for all $k \in \mathbb{N}$.

Proposition 2. For all $k \in \mathbb{N}$, we have
1. $b^{3 k}=t^{4 k} I_{3}$,
$b^{3 k+1}=t^{4 k} b$,
$b^{3 k+2}=t^{4 k} b^{2}$,
2. $b^{-3 k}=t^{-4 k} I_{3}$,
$b^{-(3 k+1)}=t^{-4(k+1)} b^{2}, \quad b^{-(3 k+2)}=t^{-4(k+1)} b$.

Proof. We prove the proposition using mathematical induction principle. Direct computations show

$$
\begin{aligned}
& b^{0}=I_{3}, b^{1}=b, b^{2}=b^{2} \\
& b^{3}=t^{4} I_{3}, b^{4}=t^{4} b, b^{5}=t^{4} b^{2} \\
& b^{-1}=t^{-4} b^{2}, b^{-2}=t^{-4} b, b^{-3}=t^{-4} I_{3} \\
& b^{-4}=\left(t^{-4}\right)^{2} b^{2}, b^{-5}=\left(t^{-4}\right)^{2} b
\end{aligned}
$$

Suppose that it is true for all integers less than or equal to $k$. We now show it is still true for $k+1$. We show (1).

$$
\begin{aligned}
& b^{3(k+1)}=b^{3 k} b^{3}=t^{4 k} I_{3} t^{4} I_{3}=t^{4(k+1)} I_{3} \\
& b^{3(k+1)+1}=b^{3 k} \cdot b^{4}=t^{4 k} I_{3} \cdot t^{4} b=t^{4(k+1)} b \\
& b^{3(k+1)+2}=b^{3 k} \cdot b^{5}=t^{4 k} I_{3} \cdot t^{4} b^{2}=t^{4(k+1)} b^{2}
\end{aligned}
$$

As for (2):

$$
\begin{aligned}
& b^{-3(k+1)}=b^{-3 k} b^{-3}=t^{-4 k} I_{3} t^{-4} I_{3}=t^{-4(k+1)} I_{3} \\
& b^{-(3(k+1)+1)}=b^{-3 k} \cdot b^{-4}=t^{-4 k} I_{3} \cdot t^{-8} b^{2}=t^{-4((k+1)+1)} b^{2}
\end{aligned}
$$




$$
b^{-(3(k+1)+2)}=b^{-3 k} \cdot b^{-5}=t^{-4 k} I_{3} \cdot t^{-8} b=t^{-4((k+1)+1)} b
$$

Lemma 1. For any non-zero integer $n, a^{n}$ and $b^{n}$ do not belong to the kernel of the reduced burau representation $B_{4} \rightarrow$ $G L\left(3, \mathbb{Z}\left[t, t^{-1}\right]\right)$.

Proof. Consider the matrix $J=\left(\begin{array}{ccc}0 & 0 & -1 \\ 1 & 0 & -1 \\ 0 & 1 & -1\end{array}\right)$. Direct computations show that

$$
J^{2}=\left(\begin{array}{lll}
0 & -1 & 1 \\
0 & -1 & 0 \\
1 & -1 & 0
\end{array}\right) \text { and } J^{3}=\left(\begin{array}{ccc}
-1 & 1 & 0 \\
-1 & 0 & 1 \\
-1 & 0 & 0
\end{array}\right)
$$

Let $n \in \mathbb{Z}$, so $n$ has one of the forms

$$
\pm 4 k, \pm(4 k+1), \pm(4 k+2) \text { and } \pm(4 k+3), \text { where } k \in \mathbb{Z} .
$$

This implies, by Proposition 1, that $a^{n}$ has to be one of the following words:

$$
t^{4 k} I_{3}, t^{4 k+1} J, t^{4 k+2} J^{2}, t^{4 k+3} J^{3} t^{-4 k} I_{3}, t^{-(4 k+1)} J^{3}, t^{-(4 k+2)} J^{2}, t^{-(4 k+3)} J .
$$

We denote by $J_{i i}^{k}$ the diagonal entry of the matrix $J^{k}$, which lies in the ith row and in the ith column $(1 \leq i \leq 3,1 \leq k \leq 3)$. Since $J_{11}, J_{11}^{2}$ and $J_{22}^{3}$ are zeros, it follows that $a^{n}$ is not the empty word for any integer $n$.

On the other hand, $n$ is written in either one of the following forms:

$$
\pm 3 k, \pm(3 k+1), \pm(3 k+2), \text { where } k \in \mathbb{Z}
$$

This implies, by Proposition 2, that $b^{n}$ has to be one of the following words:

$$
t^{4 k} I_{3}, t^{4 k} b, t^{4 k} b^{2}, t^{-4 k} I_{3}, t^{-4(k+1)} b^{2}, t^{-4(k+1)} b
$$

Direct computations show that

$$
b=\left(\begin{array}{ccc}
0 & 0 & -t \\
-t^{2} & t & -t \\
0 & t & -t
\end{array}\right) \text { and } b^{2}=\left(\begin{array}{ccc}
0 & -t^{2} & t^{2} \\
-t^{3} & 0 & t^{3} \\
-t^{3} & 0 & 0
\end{array}\right)
$$

The 1-1 entries in both of the matrices $b$ and $b^{2}$ are equal to zeros, it follows that $b^{n}$ is not the empty word for any integer $n$.

\section{Words of The Form $a^{i} b^{j}$ And $a^{i} b^{j} a^{k}$}

In this section, we use Mathematica to excute a program that computes words of the form $a^{i} b^{j}$ and $a^{i} b^{j} a^{n}$ for all non zero integers $i, j$ and $n$. In order to excute this program, we consider the following notations:

$$
\begin{array}{lrrrr}
c_{1}=a^{4 k_{1}}, & c_{2}=a^{4 k_{2}+1}, & c_{3}=a^{4 k_{3}+2}, & c_{4}=a^{4 k_{4}+3}, \\
c_{5}=a^{-4 k_{5}}, & c_{6}=a^{-\left(4 k_{6}+1\right)}, & c_{7}=a^{-\left(4 k_{7}+2\right)}, & c_{8}=a^{-\left(4 k_{8}+3\right)}, \\
z_{1}=a^{4 x_{1}}, & z_{2}=a^{4 x_{2}+1}, & z_{3}=a^{4 x_{3}+2}, & z_{4}=a^{4 x_{4}+3}, \\
z_{5}=a^{-4 x_{5}}, & z_{6}=a^{-\left(4 x_{6}+1\right)}, & z_{7}=a^{-\left(4 x_{7}+2\right)}, & z_{8}=a^{-\left(4 x_{8}+3\right)}, \\
d_{1}=b^{3 s_{1}}, & d_{2}=b^{3 s_{2}+1}, & d_{3}=b^{3 s_{3}+2}, & d_{4}=b^{-3 s_{4}}, \\
d_{5}=b^{-\left(3 s_{5}+1\right)}, & d_{6}=b^{-\left(3 s_{6}+2\right)}, & & \\
L=\left\{c_{1}, c_{2}, c_{3}, c_{4}, c_{5}, c_{6}, c_{7}, c_{8}\right\}, S=\left\{d_{1}, d_{2}, d_{3}, d_{4}, d_{5}, d_{6}\right\} & \text { and } R=\left\{z_{1}, z_{2}, z_{3}, z_{4}, z_{5}, z_{6}, z_{7}, z_{8}\right\} .
\end{array}
$$

Then we excute the following codes: 


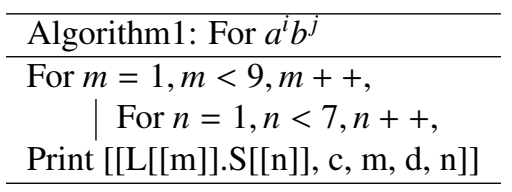

and

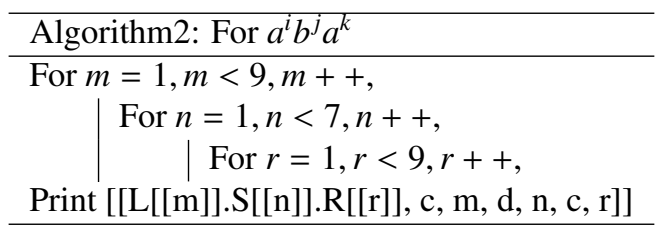

These codes compute all words of the form $a^{i} b^{j}$ and $a^{i} b^{j} a^{k}$ for all non zero integers $i, j$ and $k$. Among these words, the ones that might possibly belong to the kernel of the representation are those which have the form $t^{\alpha} I_{3}$. More precisely, these words are

$c_{1} d_{4}, c_{5} d_{1}, c_{5} d_{4}, c_{1} d_{1} z_{5}, c_{1} d_{4} z_{1}, c_{1} d_{4} z_{5}, c_{2} d_{1} z_{6}, c_{2} d_{4} z_{4}, c_{2} d_{4} z_{6}, c_{3} d_{1} z_{7}, c_{3} d_{4} z_{3}$,

$c_{3} d_{4} z_{7}, c_{4} d_{1} z_{8}, c_{4} d_{4} z_{2}, c_{4} d_{4} z_{8}, c_{5} d_{1} z_{1}, c_{5} d_{1} z_{5}, c_{5} d_{4} z_{1}, c_{5} d_{4} z_{5}, c_{6} d_{1} z_{2}, c_{6} d_{1} z_{8}, c_{6} d_{4} z_{2}$,

$c_{6} d_{4} z_{8}, c_{7} d_{1} z_{7}, c_{7} d_{4} z_{3}, c_{7} d_{4} z_{7}, c_{8} d_{1} z_{4}, c_{8} d_{1} z_{6}, c_{8} d_{4} z_{4}, c_{8} d_{4} z_{6}$.

It is clear that each of these words is the empty word. For example, $c_{1} d_{1} z_{5}=t^{\alpha} I_{3}$, where $\alpha=4 k_{1}+4 s_{1}-4 x_{5}$. If $\alpha=0$, then $x_{5}=k_{1}+s_{1}$.

Since $a^{4} \in Z\left(B_{4}\right)$ and $a^{4}=b^{3}$, it follows that $c_{1} d_{1} z_{5}=a^{4 k_{1}} b^{3 s_{1}} a^{-4 x_{5}}=a^{4 k_{1}} b^{3 s_{1}} a^{-4\left(k_{1}+s_{1}\right)}=1$.

Therefore we get the following theorem.

Theorem 3. For integers $i, j$, $k$, there are no words of the form $a^{i} b^{j} a^{k}$ which lies in the kernel of the Burau representation.

\section{References}

Artin, E. (1965). The Collected Papers of Emil Artin, Addison-Wesley Publishing Company, Inc. http://dx.doi.org/10.1007/978-1-4612-5717-2

Burau, W. (1936). Uber Zopfgruppen und gleichsinnig verdrillte Verkettungen, Abh. Math. Sem. Hamburg, 11, 179-186. http://dx.doi.org/10.1007/bf02940722

Bigelow, S. (1999). The Burau representation of the braid group $B_{n}$ is not faithful for $\mathrm{n}=5$. Geometry and Topology, 3, 397-404. http: //dx.doi.org/10.2140/gt.1999.3.397

Holtzman, C. (2008). Sous groupes de petit indice des groupes de tresses et systeme de reecriture, Doctoral thesis, institut de mathematiques de bourgogne.

Long, D ., \& Paton, M. (1992). The Burau representation of the braid group $B_{n}$ is not faithful for $\mathrm{n} \geq 6$. Topology, 32, 439-447. http://dx.doi.org/10.1016/0040-9383(93)90030-y

Magnus, W. \& Peluso, A. (1969). On a theorem of V. I. Arnold. Comm. Pure Appl. Math, 22, 683-692. http://dx.doi.org/10.1002/cpa.3160220508

Moody, J. (1991). The Burau representation of the braid group $B_{n}$ is not faithful for large n. Bull. Amer. Math.Soc., 25, 379-384. http://dx.doi.org/10.1090/S0273-0979-1991-16080-5

\section{Copyrights}

Copyright for this article is retained by the author(s), with first publication rights granted to the journal.

This is an open-access article distributed under the terms and conditions of the Creative Commons Attribution license (http://creativecommons.org/licenses/by/3.0/). 